\title{
Thermal Conductivity of Composites Under Different Heating Scenarios
}

\author{
H.T. Banks, J.H. Hogan ${ }^{\dagger}$ R.E. Tirpak ${ }^{\dagger}$, S. Wynne*
}

October 5, 2003

\begin{abstract}
We study the overall thermal conductivity of composites under three different heating scenarios: (i) a laser pulse heat source, (ii) a preheated composite sample, and (iii) a continuous heat source.
\end{abstract}

\section{Introduction}

Adhesives such as epoxies, gels, and greases have numerous commercial and industrial applications, such as computers, machinery, and home appliances. These adhesives are typically very poor conductors. Hence, filler particles, with higher thermal conductivities, such as diamond dust, carbon fibers, or aluminum particles, are added to create a composite material that is a better thermal conductor than the original material. The filler particles introduce several design considerations, including the choice of particle, the particle geometry, and the size and shape of the particles.

In [1] the authors present a mathematical model to describe the two dimensional heat transfer through a composite silicone. The physical model involves a thin slice of composite material with a pulsed heat source (laser) at one end, a heat sink at the other end (ambient air), and no heat loss through the sides. See Figure 1 for a depiction of the physical model. The corresponding mathematical model is based on the transient heat equation with insulated boundary conditions on the sides, a flux due to the heat source at one end, and Newton cooling at the other end. Let $u(t, z)$ represent the composite silicone temperature at a given time $t$ and coordinate $z$. Let $k(z)$,

${ }^{*}$ Center for Research in Scientific Computation, Box 8205, North Carolina State University, Raleigh, NC 27695-8205

${ }^{\dagger}$ Lord Corp.,406 Gregson Dr., Cary, NC 27511 


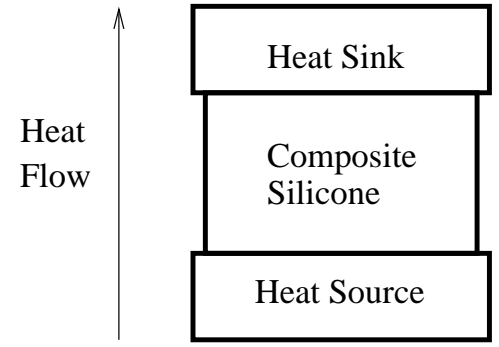

Figure 1: Two dimensional heat transfer model

$\rho(z)$, and $c_{p}(z)$ represent the thermal conductivity, density, and specific heat, respectively, of the composite at coordinate $z$. Then the following system describes the temperature in the composite sample for $z \in \Omega=$ $\left[-\frac{c_{1}}{2}, \frac{c_{1}}{2}\right] \times\left[-\frac{c_{2}}{2}, \frac{c_{2}}{2}\right]$ and $t \in[0, T]$,

$$
\begin{aligned}
& \rho(z) c_{p}(z) \frac{\partial u}{\partial t}(t, z)=\nabla \cdot(k(z) \nabla u(t, z)), \quad u(0, z)=u_{0}(z), \quad z \in \Omega \\
& \left.k(z) \frac{\partial u}{\partial n}(t, z)\right|_{\gamma_{1}}=0 \\
& \left.k(z) \frac{\partial u}{\partial n}(t, z)\right|_{\gamma_{2}}=\left.h\left(T_{\infty}-u(t, x)\right)\right|_{\gamma_{2}} \\
& \left.k(z) \frac{\partial u}{\partial n}(t, z)\right|_{\gamma_{3}}=0 \\
& \left.k(z) \frac{\partial u}{\partial n}(t, z)\right|_{\gamma_{4}}=S_{0}(t) .
\end{aligned}
$$

Here $T_{\infty}$ denotes the ambient temperature, $h$ is the Newton cooling constant (or heat transfer coefficient), $S_{0}(t)$ is the flux due to the heat source, and $u_{0}$ is the initial temperature distribution in the composite silicone. The boundaries are described by the parameterized $\gamma_{i}$, where

$$
\begin{aligned}
\gamma_{1}(s)=\left(\frac{c_{1}}{2}, s\right): s \in\left[-\frac{c_{2}}{2}, \frac{c_{2}}{2}\right], & \gamma_{2}(s)=\left(s, \frac{c_{2}}{2}\right): s \in\left[-\frac{c_{1}}{2}, \frac{c_{1}}{2}\right], \\
\gamma_{3}(s)=\left(-\frac{c_{1}}{2}, s\right): s \in\left[-\frac{c_{2}}{2}, \frac{c_{2}}{2}\right], & \gamma_{4}(s)=\left(s,-\frac{c_{2}}{2}\right): s \in\left[-\frac{c_{1}}{2}, \frac{c_{1}}{2}\right],
\end{aligned}
$$

and $\partial \Omega=\gamma_{1} \cup \gamma_{2} \cup \gamma_{3} \cup \gamma_{4}$. See Figure 2 for a depiction of the two dimensional slice with indicated boundaries. Let $\Omega_{s}$ denote the region occupied by the silicone and $\Omega_{p}$ denote the region occupied by the filler particles. Then $\Omega=$ $\Omega_{s} \cup \Omega_{p}$ and $\emptyset=\Omega_{s} \cap \Omega_{p}$. Note that the coefficients of the partial differential equation, $k(z), \rho(z)$, and $c_{p}(z)$, are piecewise constant. For example, $k(z)=$ $k_{s}$ for $z \in \Omega_{s}$ and $k(z)=k_{p}$ for $z \in \Omega_{p}$. 


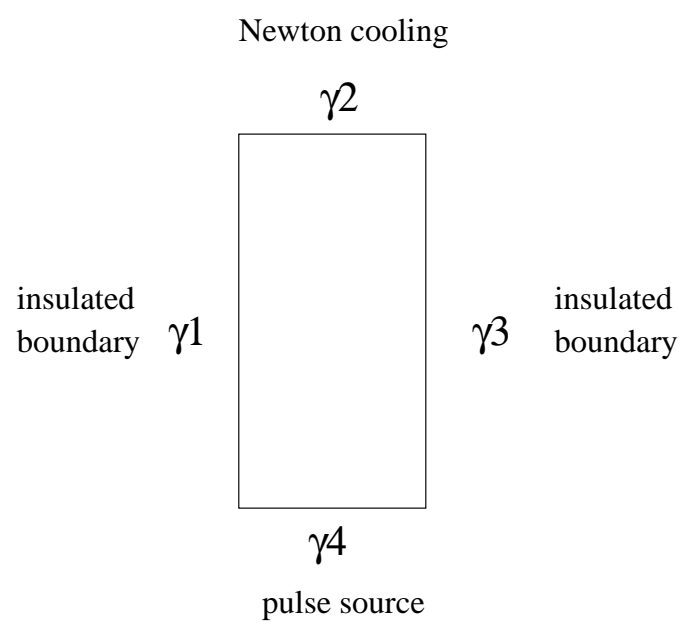

Figure 2: 2D composite adhesive slice

In [1] the authors show this model is well-posed, i.e., there exist unique weak solutions which are continuously dependent on the initial conditions, forcing function and parameters. They also develop mathematical models that describe the sensitivity of temperature on the silicone and filler particle thermal conductivities and establish the well-posedness of the sensitivity models. Their computational investigations involve a uniform arrangement of circular filler particles within the silicone. From these investigations, the authors conclude that the model is not very sensitive to filler particle conductivity.

In this note we continue the computational investigations of [1] to further understand the heat transfer through a composite silicone. We concentrate our presentation on a composite material of fixed size and consider the effect of particle size and arrangement. Our investigations explore three experimental scenarios. The first scenario involves a laser pulse heat source as described in [1] and above. This scenario imitates a heat surge above a steady state condition. Solutions for this scenario are highly transient, particularly with respect to the sensitivity analysis. Hence, for a second scenario we consider an initially heated adhesive with three insulated boundaries and the fourth boundary exposed to cooling (ambient air). The third scenario involves a continuous heat source and focuses on steady state solutions. This scenario imitates a situation wherein silicone in a computer motherboard is in the presence of continuous power source such as during regular computer 
usage.

The numerical experiments are outlined below. In Section 2 we report on experiments with a laser pulse heat source. Much of the detail and discussion is included in this first to better understand the basic heat transfer behavior. In Sections 3 and Section 4 we consider the preheated and continuous heat source scenarios, respectively. The presentations in these later sections are similar to Section 2 and their results are intended for comparison. Some conclusions are summarized in Section 5.

Our numerical results will show that, in general, a smaller particle size and larger volume fraction can slightly improve the heat transfer through composite adhesives. However, larger gains may be realized by increasing the silicone thermal conductivity in the case of a continuous or pulsed heat source.

\section{Pulse Heat Source Experiments}

We consider the basic model presented above, i.e., insulated boundaries on the sides, a laser pulse heat source on one end, and Newton cooling on the other end. The size of the adhesive sample is $107.67 \times 546$, i.e., $c_{1}=107.67 \mu$ and $c_{2}=546 \mu$. The laser pulse heat source is a flux term of the form

$$
S_{0}(t)= \begin{cases}S_{l} & 0 \leq t \leq t_{p} \\ 0 & t_{p}<t\end{cases}
$$

where $t_{p}=0.000330$ seconds. The model is numerically solved on a triangular mesh generated by the Matlab PDE Toolbox using the Delaunay triangulation algorithm. We integrate system (1) on the interval $t \in[0,3.46]$ seconds using a finite element method from Matlab's PDE Toolbox. See [1] for details about the source flux or the use of the PDE Toolbox.

In working with transient solutions (i.e., a pulse source) the temperature profiles, flux, and in particular, sensitivity results, are highly time dependent. Additionally, the time frame for rapid changes is affected by the silicone thermal conductivity. To better understand the basic heat transfer behavior we first look at an adhesive sample without particles. The adhesive will have the material characteristics of either silicone or filler particle. We then look at filled composite adhesives, with either a uniform or random particle arrangement. Finally, we consider the effect of particle size, placement and volume fraction on heat transfer. 


\subsection{Without Particles}

To investigate the effect of changing the thermal conductivity, we consider an unfilled adhesive sample. We work with two cases: a sample with thermal conductivities in the silicone range $(k=0.02-1.02 \mathrm{~W} / \mathrm{mK})$, and a sample with thermal conductivities in the filler particle range $(k=$ $100-1100 \mathrm{~W} / \mathrm{mK})$. The density and specific heat parameters are set according to the material in each case. The model parameters are listed in Table 1.

\begin{tabular}{|c|c|c|c|c|c|}
\hline$k(W / m K)$ & $\rho\left(g / \mathrm{cm}^{3}\right)$ & $c_{p}(J / g K)$ & $T_{\infty}(K)$ & $h$ & $S_{l}\left(W / m^{2}\right)$ \\
\hline $0.02-1.02$ & $1 g$ & 1.55 & 296.15 & 350 & $4.32 x 10^{7}$ \\
\hline $100-1100$ & 2.7 & 0.90 & 296.15 & 350 & $4.32 x 10^{7}$ \\
\hline
\end{tabular}

Table 1: Model parameters

In Figure 3 we plot the temperature in the sample at various times during the time integration for two different silicone thermal conductivities. The first column of plots indicate temperatures for a sample with $k=0.02 \mathrm{~W} / \mathrm{mK}$; the second column indicates temperatures for a sample with $k=1.02 \mathrm{~W} / \mathrm{mK}$. At $t=0.0115$ seconds, the pulse may be clearly identified in one end of the slice. After $t=1.1418$ seconds, the pulse is distributed through the slice, and at the end of the time integration $(t=3.46 s)$, most of the heat is convected away by the Newton cooling boundary condition. Note the difference in temperature range for a slice with low $(0.02 \mathrm{~W} / \mathrm{mK})$ versus high $(1.02 \mathrm{~W} / \mathrm{mK})$ silicone thermal conductivity. Increasing the silicone thermal conductivity results in a large improvement in heat transfer.

We next consider a sample with thermal conductivities in the range of filler particles. In Figure 4 we plot the temperature in the sample at various times for two different thermal conductivities. The first column plots temperatures with $k=100 \mathrm{~W} / \mathrm{mK}$; the second column plots temperatures with $k=1100 \mathrm{~W} / \mathrm{mK}$. In each case, after only $t=0.0115$ seconds, much of the pulse has moved through the sample. There is relatively little difference in temperature between plots with low and high particle thermal conductivity. Hence, in this experiment, changing the particle conductivity alone does not greatly affect the resulting heat transfer within the sample. Note the difference in temperature ranges for a particle sample (Figure 4) versus a silicone sample (Figure 3).

Next we compute the average temperature in a slice at a particular 

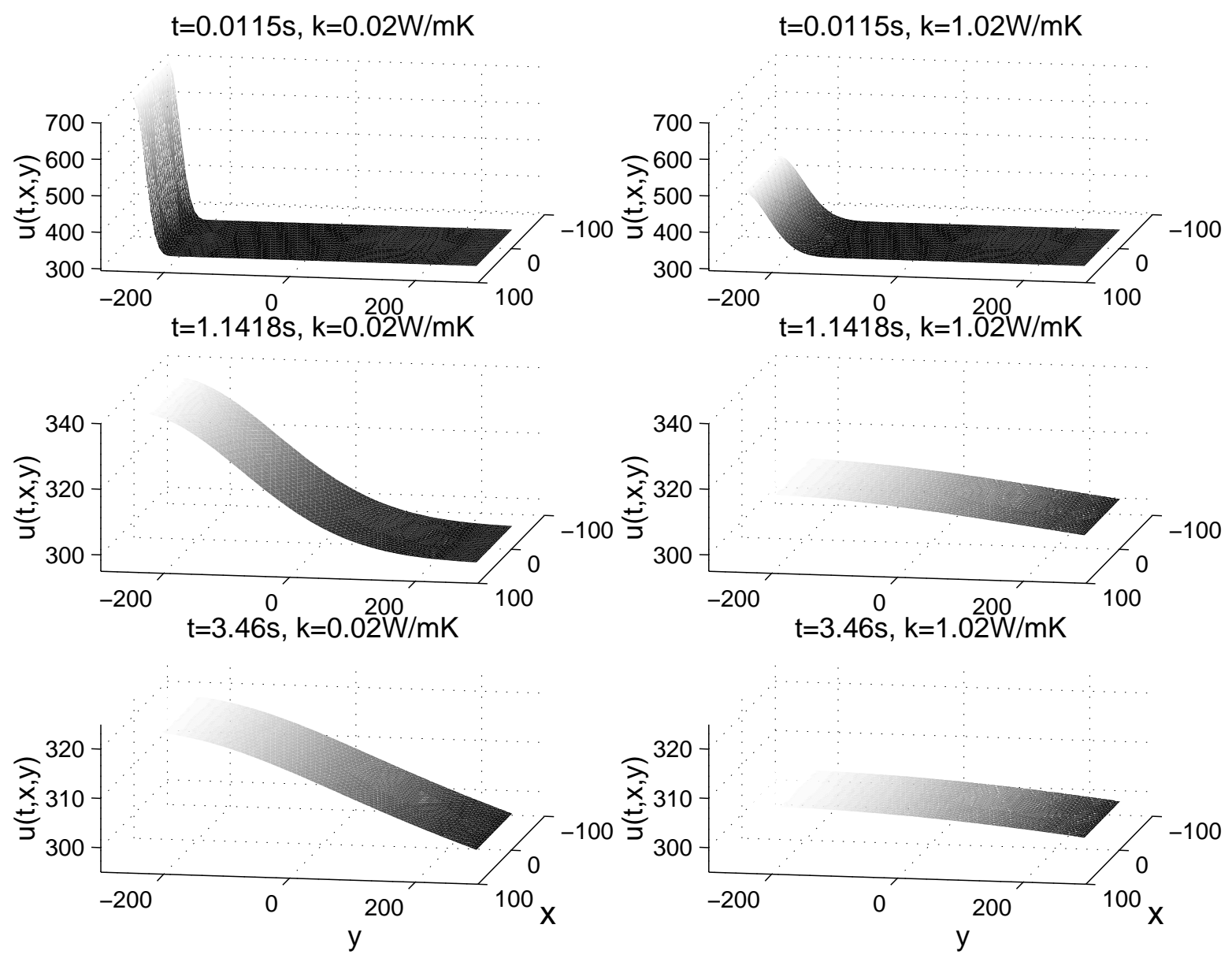

Figure 3: Silicone sample 


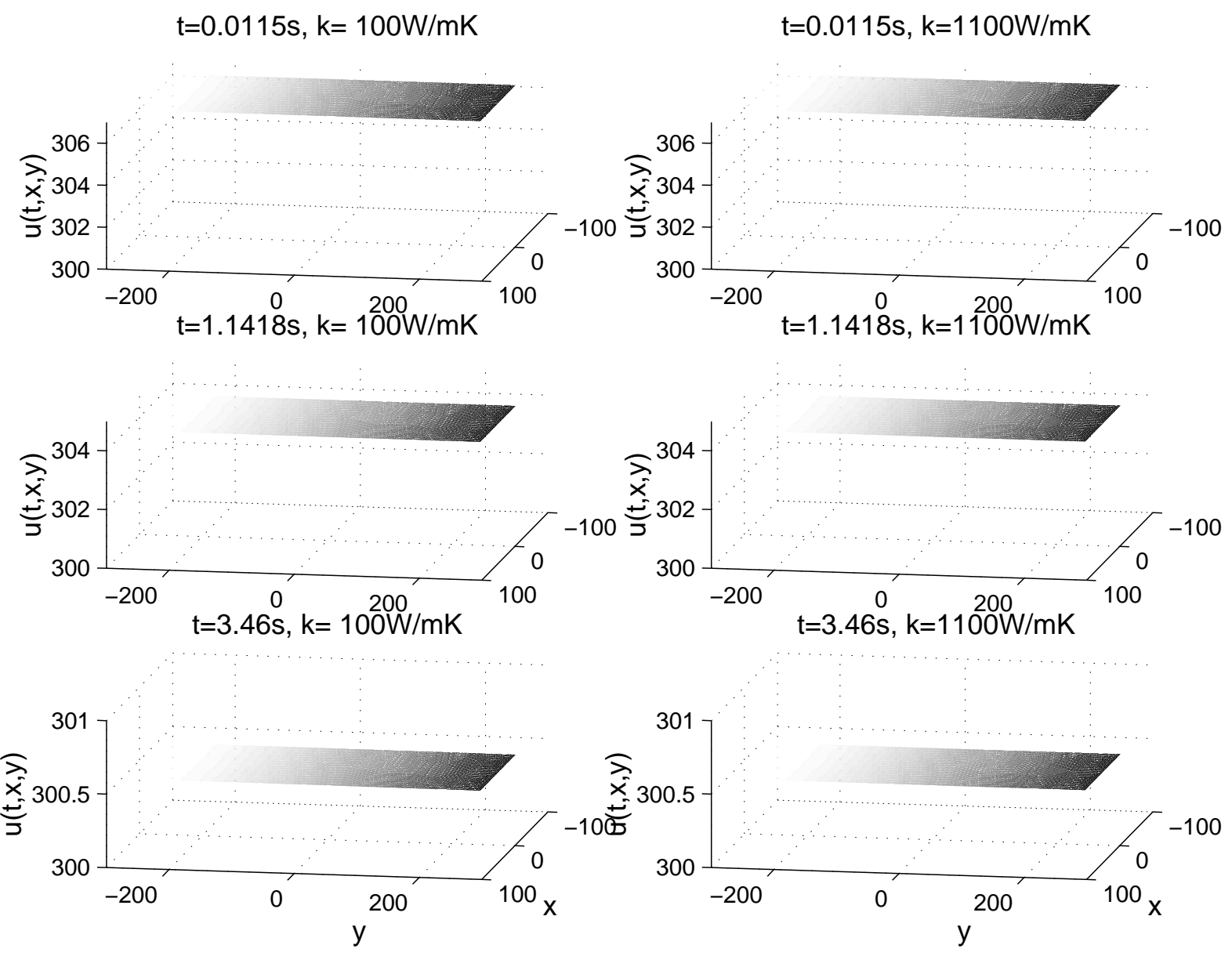

Figure 4: Filler particle sample 

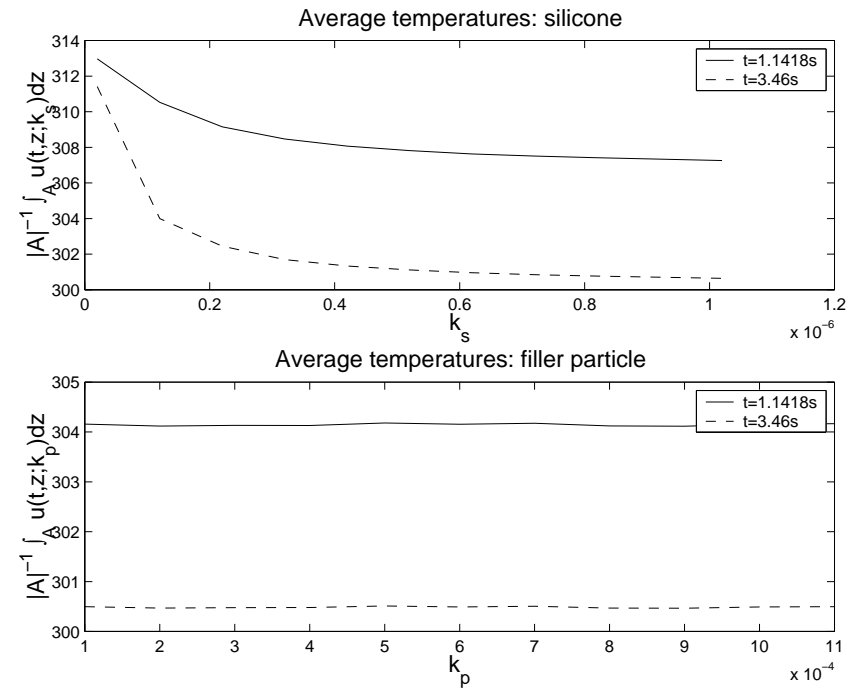

Figure 5: Average temperatures at $t=1.14 \mathrm{~s}$ and $t=3.46 \mathrm{~s}$.

time and compare average temperatures over a range of adhesive thermal conductivities. We compute the average temperature as follows

$$
|A|^{-1} \int_{A} u(t, z ; k) d z
$$

where $A$ represents the area of the adhesive. In Figure 5 we plot the average temperature in the slice for $k$ in the range of silicone conductivities, and for $k$ in the range of filler particle conductivities. The solid line represents average temperatures at $t=1.1418$ seconds into the time integration; the dashed line represents average temperatures at the final time $t=3.46$ seconds. Recall that the ambient temperature is $T_{\infty}=296.15 K$. Clearly, increasing silicone conductivity is more effective in lowering the average temperature in the slice, though the effect levels off at higher silicone conductivities. Increasing particle conductivity has no visible effect.

The basic heat transfer mechanisms present are conduction and convection. Heat is transfered through the slice by conduction; heat is transfered from the slice to the ambient air by convection due to the Newton cooling boundary condition at $\gamma_{2}$. From the boundary condition, the flux at $\gamma_{2}$ is proportional to the temperature gradient at $\gamma_{2}$. In Figure 6 we plot the flux at $\gamma_{2}$, averaged over $\gamma_{2}$, as a function of time for various thermal 

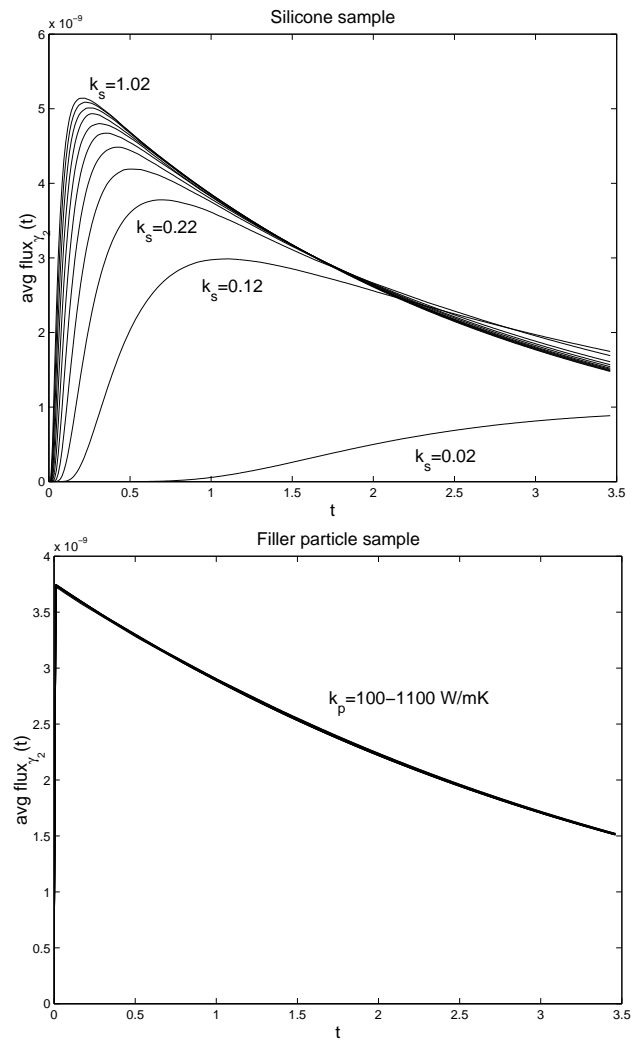

Figure 6: Average flux at $\gamma_{2}$.

conductivities. Specifically, we plot

$$
\overline{-k(z) \frac{\partial u}{\partial n}(t, z)}^{\gamma}=-k\left|\gamma_{2}\right|^{-1} \int_{\gamma_{2}} \frac{\partial u}{\partial y}(t, s) d s .
$$

The top plot gives the average flux for thermal conductivities in the silicone range, $k=k_{s} \in[0.02,1.02] \mathrm{W} / \mathrm{mK}$; the bottom plot displays the average flux for thermal conductivities in the filler particle range, $k=k_{p} \in$ $[100,1100] \mathrm{W} / \mathrm{mK}$. For conductivities in the particle range, increasing the conductivity has little effect on the average flux at $\gamma_{2}$. Conversely, for conductivities in the silicone range, increasing the conductivity does increase the average flux at $\gamma_{2}$. This increase saturates with the larger silicone conductivities due to the Newton cooling boundary condition at $\gamma_{2}$ and the cooling rate $(h)$. 
The constant $h$ depends on several factors: the adhesive properties $(k, \mu$, $\left.\rho, C_{p}\right)$, the system geometry, the flow velocity, the value of the characteristic temperature difference at the boundary, as well as surface temperature distribution. For our experiments, the value of $h$ mimics ambient air cooling. The use of fans or other devices could result in a faster or slower cooling rate, respectively.

We now look at results from the sensitivity equations. Let $w(t, z)=$ $\frac{\partial u}{\partial k}(t, z)$ represent the sensitivity of temperature to thermal conductivity. See [1] for the sensitivity equations and their derivation. Since the temperature solutions are transient, the sensitivities are highly time-dependent. In [1] the authors plot the sensitivity at $\gamma_{2}$ (average sensitivity or relative average sensitivity) as a function of conductivity at various instances in time. Instead we plot sensitivity at $\gamma_{2}$, averaged over $\gamma_{2}$, as a function of time for various adhesive thermal conductivities. More specifically, we compute the average sensitivity

$$
w_{2}(t ; k)=\left|\gamma_{2}\right|^{-1} \int_{\gamma_{2}} w(t, s ; k) d s
$$

for $k$ in the silicone range or the filler particle range. The top plot in Figure 7 displays the average sensitivity to silicone thermal conductivity at $\gamma_{2}$; the bottom plot displays the average sensitivity to particle thermal conductivity at $\gamma_{2}$. A positive/negative average sensitivity indicates that the temperature is increasing/decreasing (on average) with respect to $k$ at $\gamma_{2}$. Recall that the slice is initially at ambient temperature. As the pulse distributes through the slice the temperature at the sink boundary $\gamma_{2}$ initially increases. Increasing the thermal conductivity has the effect of transferring more heat from the source end to the sink end, some of which is not immediately convected away and results in a higher temperature at $\gamma_{2}$. As heat is convected from the slice, temperatures eventually decrease at $\gamma_{2}$. Increasing the thermal conductivity results in more effective heat transfer to $\gamma_{2}$ and lower temperatures. For conductivities in the particle range, the decrease in temperature occurs almost immediately; for conductivities in the silicone range, temperatures initially increase and then decrease.

Note the different ranges for average sensitivity to silicone conductivity versus particle conductivity, indicating that heat transfer is more sensitive to changes in silicone conductivity than particle conductivity. Also note that for $t>1 s$, temperature is insensitive to both particle or silicone thermal conductivity (except for the lowest silicone conductivity). 

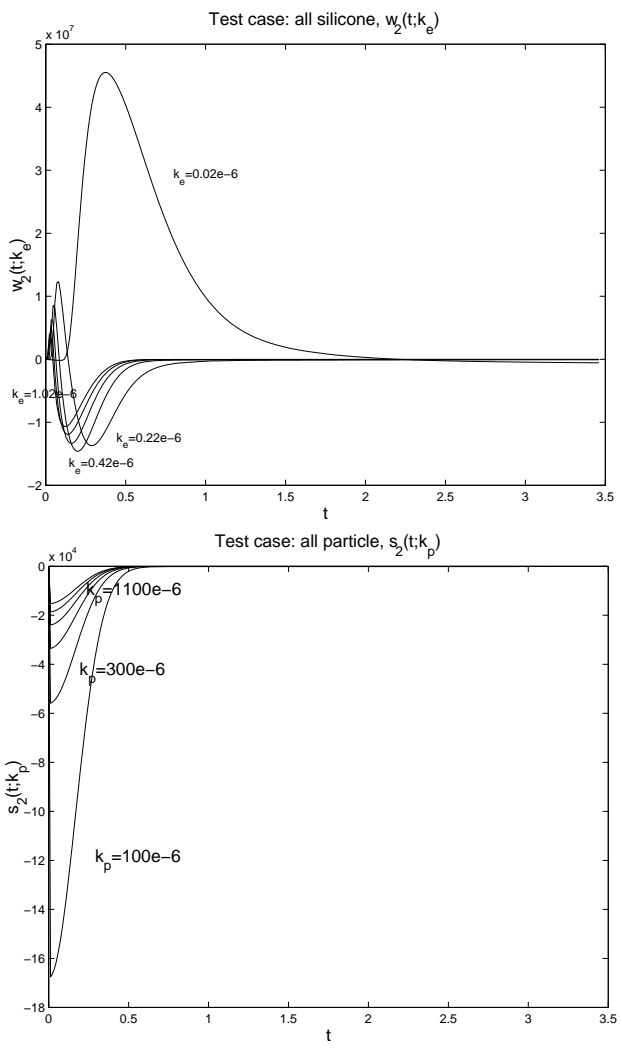

Figure 7: Average sensitivity at boundary $\gamma_{2}$.

\subsection{With Particles}

In this section we include filler particles within the adhesive. The particles are circular and uniform in size with radius $12.07 \mu$; the particles are aluminum with fixed thermal conductivity $k_{p}=217 \mathrm{~W} / \mathrm{mK}$. Since the silicone polymer is assumed to wet well, we assume a minimum distance between particles and that no particles touch the boundary of the composite silicone slice. The size of the silicone slice is the same as above.

We consider two different particle arrangements: a uniform arrangement of thirty-two particles aligned in two rows (UNI), and a random arrangement of thirty-two particles (RAND). Both arrangements constitute a volume fraction of $25 \%$. The results reported in [1] refer only to a uniform particle arrangement, however a random arrangement is more realistic. See Figure 8 for the particle arrangements. The purpose of these experiments is to 

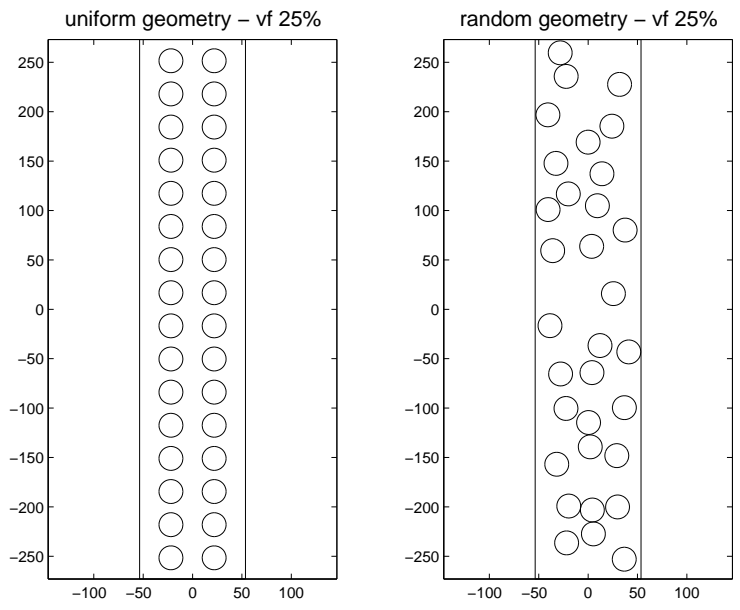

Figure 8: Composite adhesive particle arrangement vf $25 \%$

determine the effect of different particle arrangements, and also to compare with results from silicone slices in the previous subsection.

In Figure 9 we plot the temperature profiles resulting from the uniform and random particle arrangements at the intermediate time, $t=1.1418 \mathrm{~s}$. The top two plots provide the profiles for the uniform and random arrangements with a low silicone thermal conductivity $\left(k_{s}=0.02 \mathrm{~W} / \mathrm{mK}\right)$; the bottom two plots provide profiles for each arrangement with a high silicone thermal conductivity $\left(k_{s}=1.02 \mathrm{~W} / \mathrm{mK}\right)$. Again the particle thermal conductivity is set for aluminum with $k_{p}=217 \mathrm{~W} / \mathrm{mK}$. There are slight differences in profiles due to the particle arrangements (i.e., heated rows versus irregular warm spots), however, the overall temperatures for each arrangement appear similar.

In Figure 10 we plot the average temperature in the uniform composite slice (UNI), the random composite slice (RAND), and the all silicone slice (SIL) at $t=1.1418 \mathrm{~s}$ and at the final time $t=3.46 \mathrm{~s}$ for a range of silicone thermal conductivities. The average temperatures for the composite slices (UNI and RAND) are nearly identical at either time. At the intermediate time $(t=1.1418 s)$, the composite silicone slices have lower average temperatures than the all silicone slice across the range of silicone thermal conductivities. In other words, the composite slices are more effective in transferring heat through and out of the adhesive slice. This is also true at the final time $(t=3.46 s)$, however, the average temperatures saturate as 

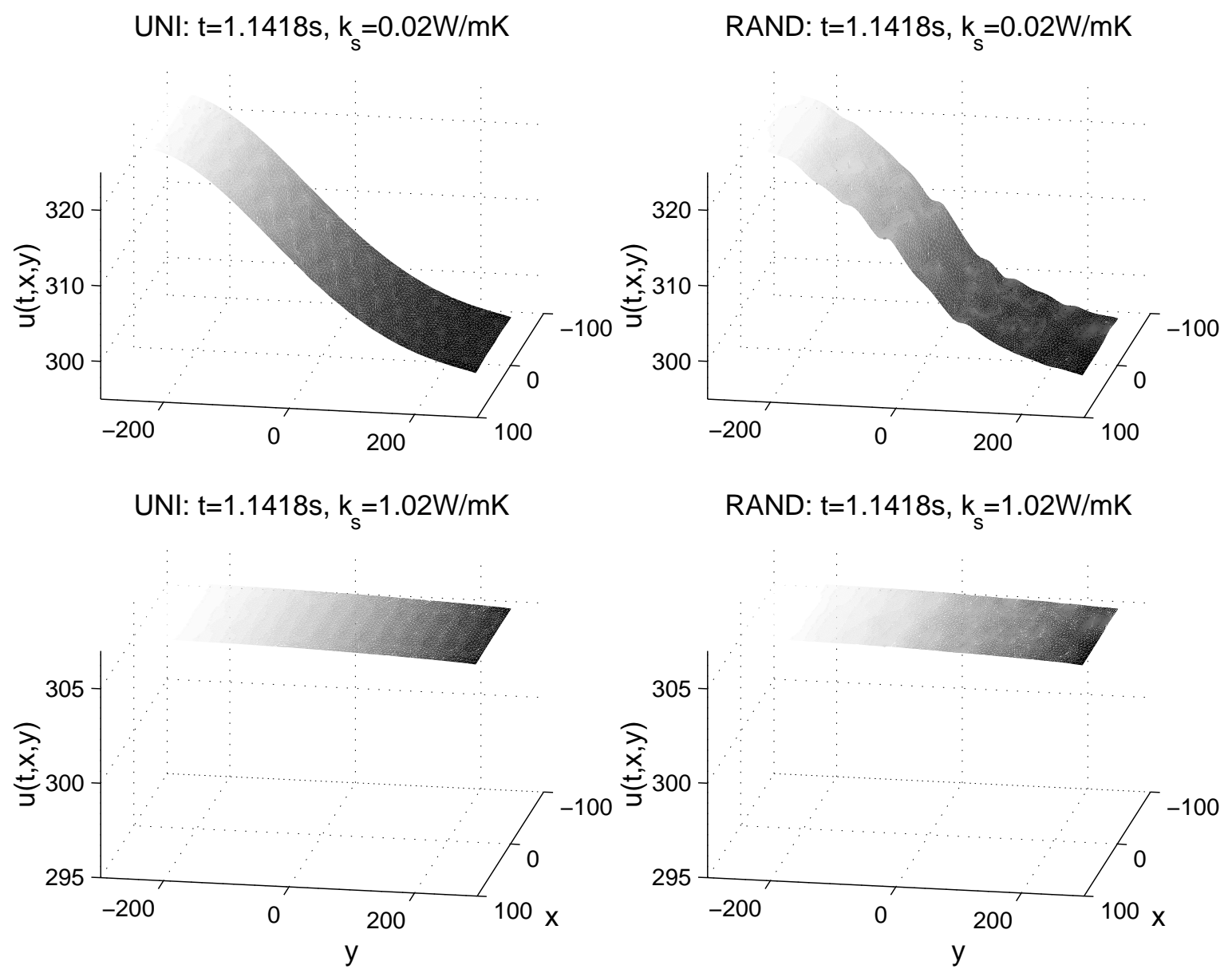

Figure 9: Temperature profiles for composite adhesives 

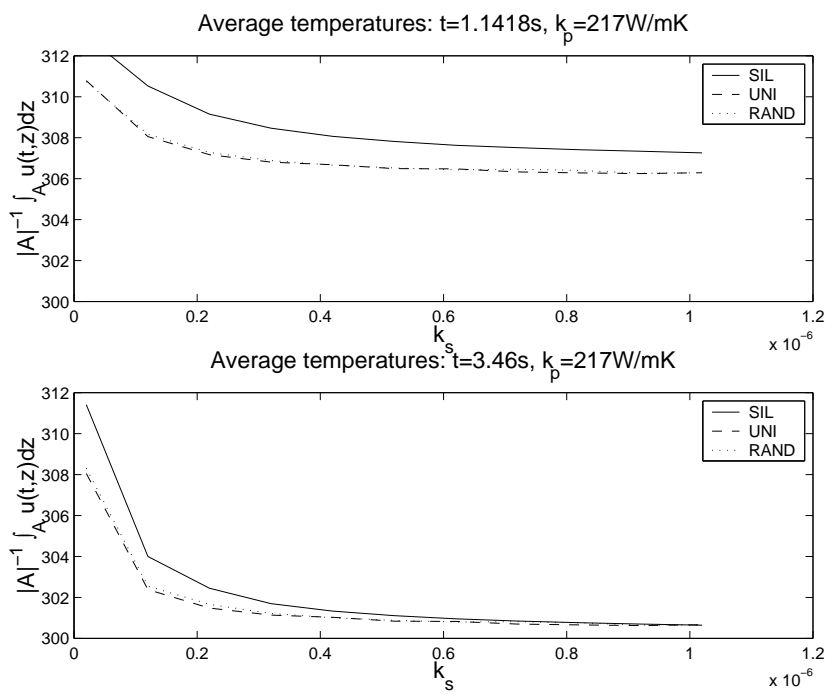

Figure 10: Average temperature for composite adhesives, vf $25 \%$

$k_{s}$ increases since most of the heat has convected away. Little improvement in heat transfer is realized by increasing the silicone thermal conductivity beyond $0.3 \mathrm{~W} / \mathrm{mK}$.

\subsection{Fixed Volume Fraction}

In the previous subsection the uniform versus random particle placement did not noticeable affect the resulting temperatures from a pulse source. In this section we investigate in more detail the effect of particle placement and particle size on heat transfer.

We fix the volume fraction at $25 \%$ and vary the size and placement of the particles. Again, we work with aluminum filler particles $\left(k_{p}=217 \mathrm{~W} / \mathrm{mK}\right)$. All slices contain a uniform particle placement: 3 slices with particles arranged in two rows, 3 slices with particles arranged in 1 row. In each case the number of particles is inversely proportional to the size of the particles. See Figure 11 for the different particle arrangements with $25 \%$ volume fraction.

To better observe trends in heat transfer, we plot the temperatures through the middle of each slice $(x=0)$ at the time $t=1.1418 s$ for two different silicone thermal conductivities in Figure 12. The top two plots give temperature profiles for a low silicone conductivity $\left(k_{s}=0.02 \mathrm{~W} / \mathrm{mK}\right)$; the bottom two plots show temperature profiles for a high silicone conductivity 

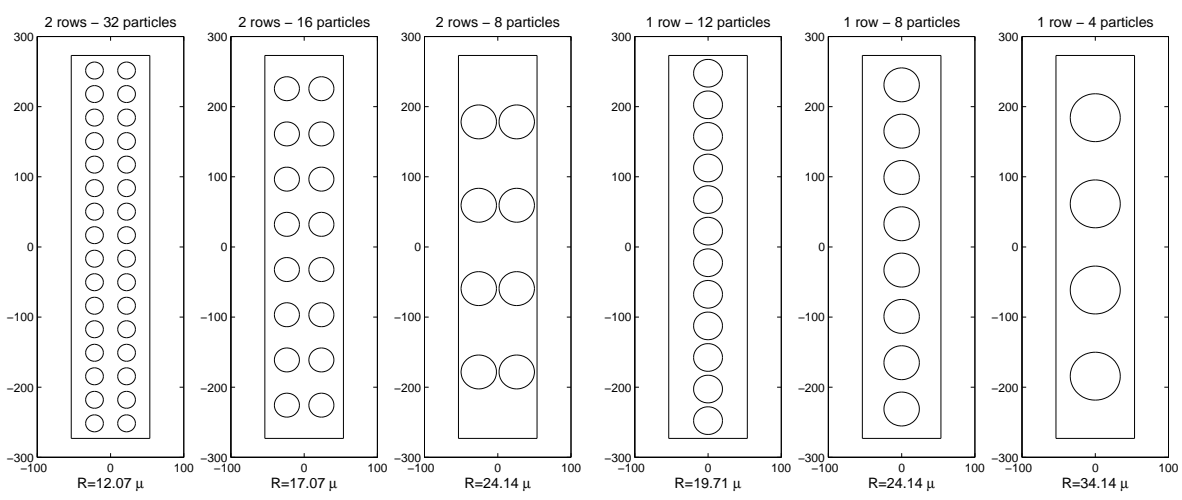

Figure 11: Uniform geometries with $25 \%$ volume fraction
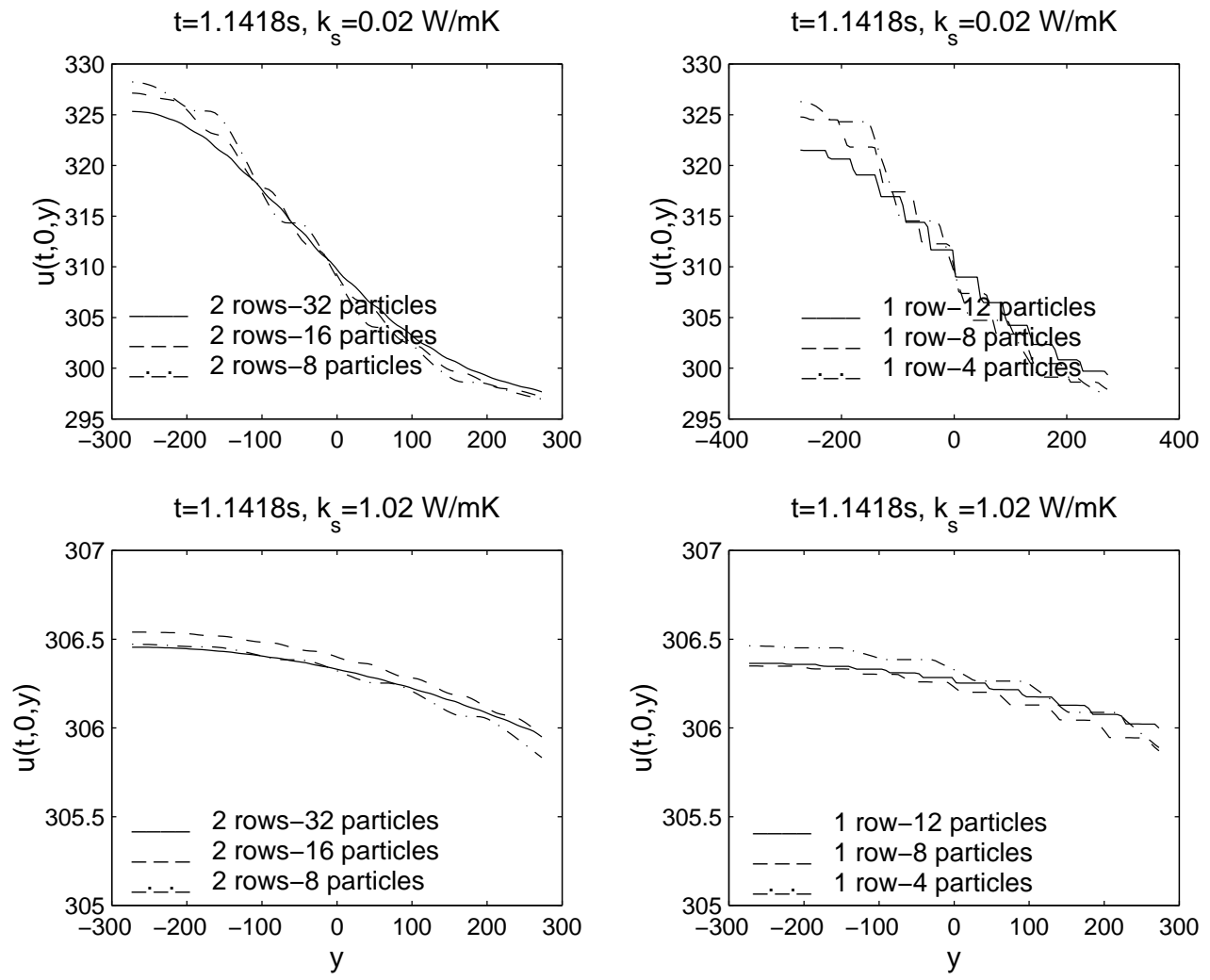

Figure 12: Temperature profiles at $x=0,25 \%$ volume fraction 


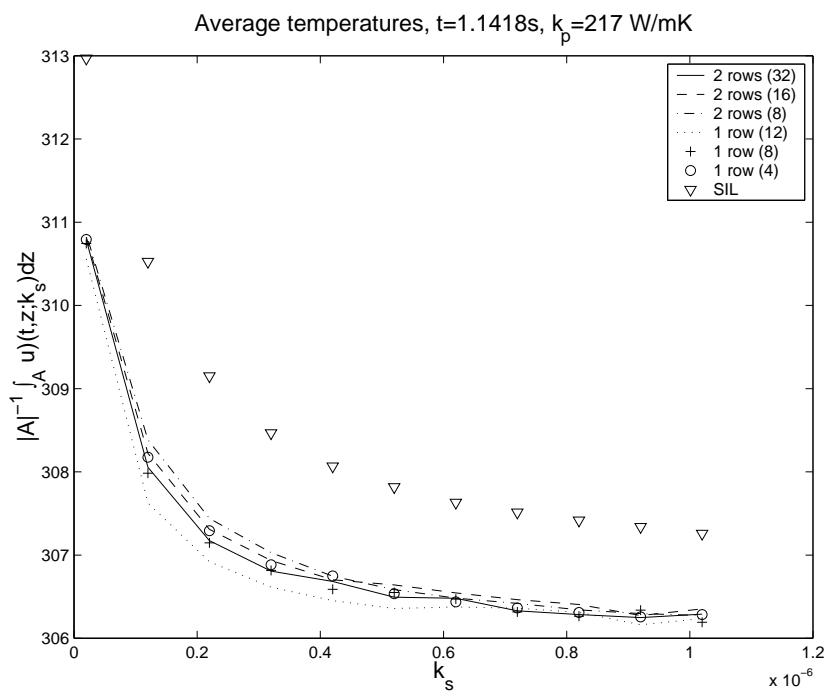

Figure 13: Average temperatures, $25 \%$ volume fraction

$\left(k_{s}=1.02 \mathrm{~W} / \mathrm{mK}\right)$. Temperatures are plotted at an intermediate time since most of the laser pulse has dissipated away by the end of the time integration. The differences in temperature profiles for each of the different uniform geometries are slight. However, the heat transfer for the eight particle case is more efficient with particles aligned in one row as opposed to two rows. Placement of the particles in one row, with the particles closer together, acts as a channel to direct the heat transfer through the filler particles.

In Figure 13 we plot the average temperature for each of the uniform particle arrangements at the time $t=1.1418 \mathrm{~s}$ across a range of silicone thermal conductivities. For reference we have also included the average temperature in the all silicone slice (SIL) at the same time across the same range of conductivities. There are slight differences in the average temperatures between the various uniform geometries of $25 \%$ volume fraction, though they all remain close together. In general the composite slices with smaller, more numerous particles produce lower average temperatures. All the composite slices obtain lower average temperatures than the silicone slice by approximately $2 K$. 


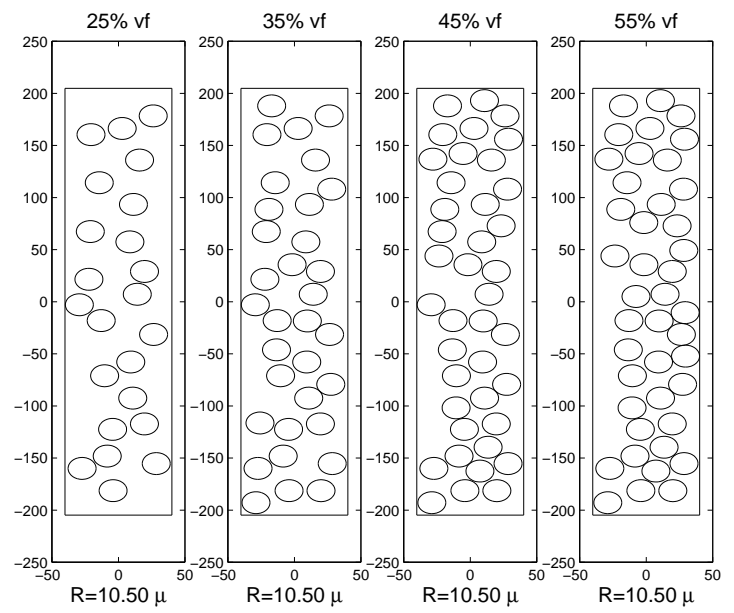

Figure 14: Random geometries with varied volume fraction

\subsection{Varied Volume Fraction}

In this section we look at the effect of volume fraction on heat transfer through a composite adhesive. Here we fix the particle size $(R=10.50 \mu)$ and vary the number of particles (volume fraction) in the composite adhesive. The particles are arranged randomly to form volume fractions of $25 \%$, $35 \%, 45 \%$, and $55 \%$. See Figure 14 for the random particle arrangements. The particles are aluminum $\left(k_{p}=217 W / m K\right)$. The size of the slice and particles are smaller than in the previous section to ease the domain generation performed by MATLAB for the high volume fraction domains. Here $c_{1}=80.375 \mu$ and $c_{2}=409.5 \mu$.

We are looking for trends in heat transfer behavior as we increase volume fraction. From the experiments with fixed volume fraction, there appears to be a slight benefit to smaller, closer particles. The expectation is that the higher the volume fraction, the more effective the heat transfer.

In Figure 15 we plot the temperature profiles through the middle of the slice $(x=0)$ at the time $t=1.1418 s$ for two different silicone thermal conductivities. The top plot displays temperature profiles for the low silicone conductivity $\left(k_{s}=0.02 \mathrm{~W} / \mathrm{mK}\right)$; the bottom plot displays temperature profiles for the high silicone conductivity $\left(k_{s}=1.02 \mathrm{~W} / \mathrm{mK}\right)$. Both plots display the same trend: larger volume fractions result in lower temperatures. The total temperature difference is less than $1 K$, however, for slices with high silicone thermal conductivity. 

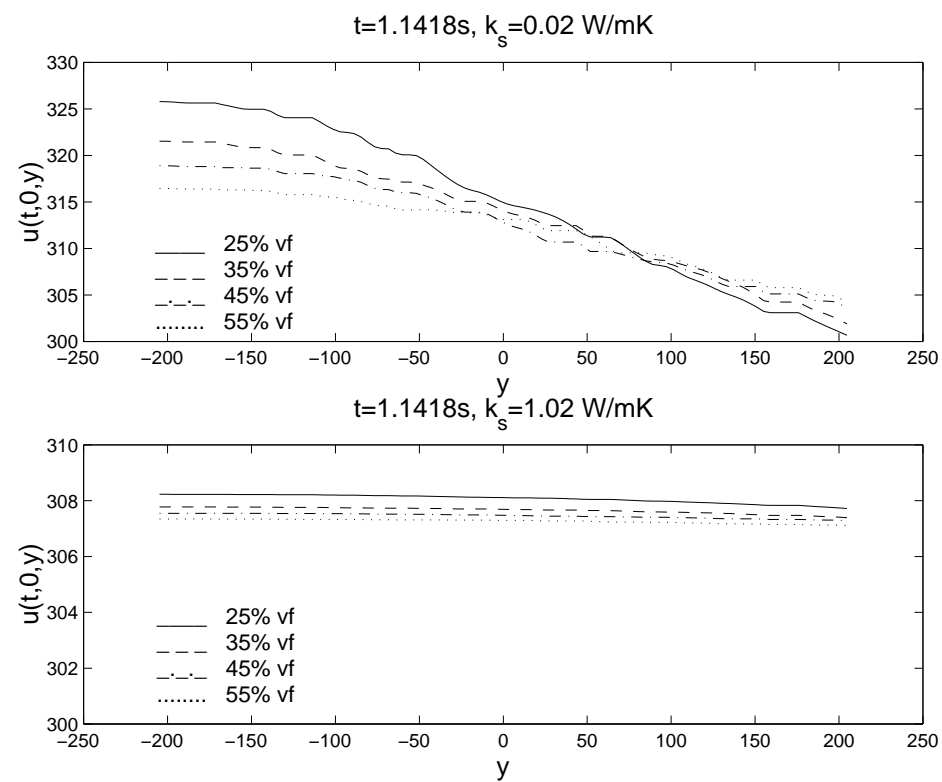

Figure 15: Temperature profiles at $x=0$, varied volume fraction

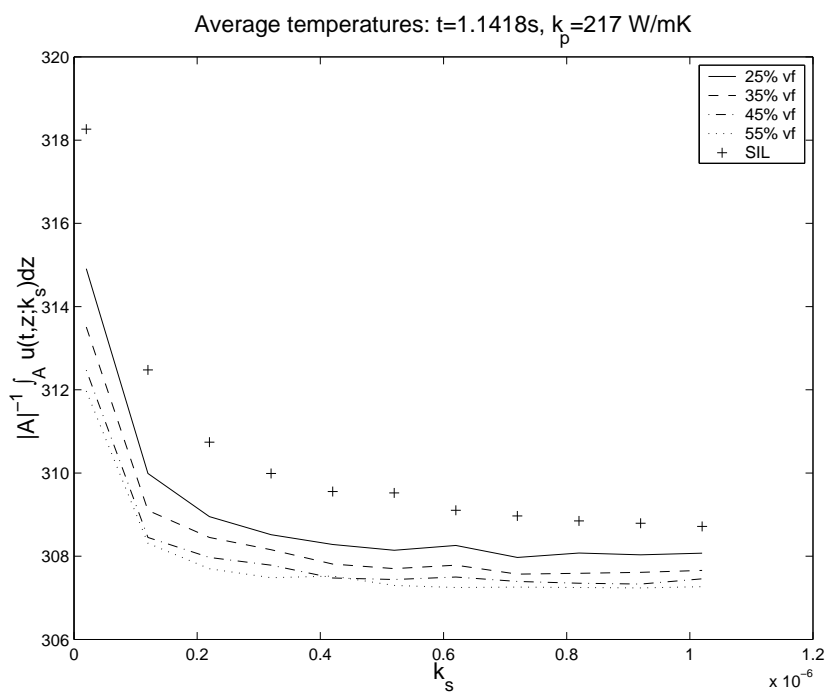

Figure 16: Average temperatures, varied volume fraction 


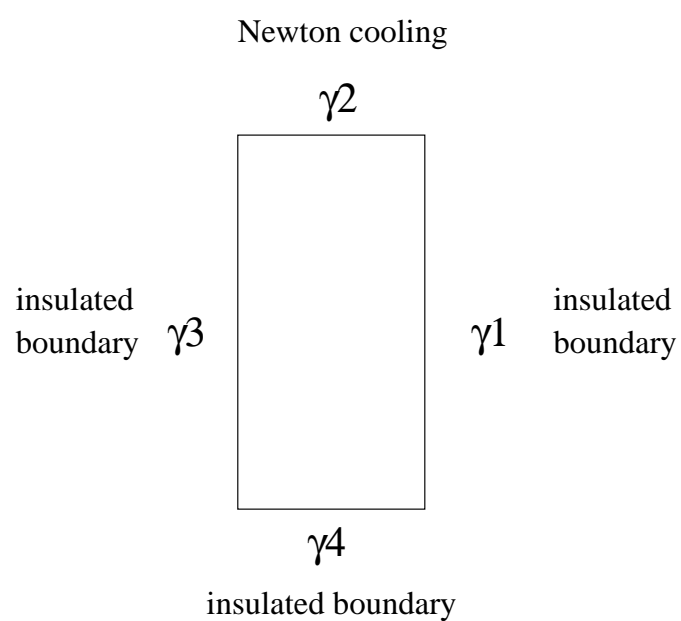

Figure 17: Initially heated 2D composite adhesive slice

This trend is more clearly seen in Figure 16 where we plot the average temperatures for each slice at the time $t=1.1418 \mathrm{~s}$ across a range of silicone thermal conductivities. Again, we include the average temperature for the all silicone slice (SIL) for the same range of silicone conductivities. All composite slices have a lower average temperature than the all silicone slice; The higher the volume fraction, the lower the average temperature. Note, however, that the difference in average temperatures from $25-55 \%$ volume fraction is only $1.5 \mathrm{~K}$. Also note that all average temperatures saturate, at $k_{s}>0.3 \mathrm{~W} / \mathrm{mK}$ for composite slices and at $k_{s}>0.7 \mathrm{~W} / \mathrm{mK}$ for the silicone slice. Prior to saturation, however, an increase in silicone thermal conductivity results in lower average temperatures. Hence, another means of improving heat transfer may be accomplished by increasing the silicone thermal conductivity up to the saturation point.

\section{$3 \quad$ Heated Slice Experiments}

In this section we work with a slightly different experimental set-up to better avoid the transient nature of solutions in the previous section. Rather than using a laser pulse heat source, the slice is pre-heated to a temperature of $650 \mathrm{~K}$. Three sides of the sample are insulated $\left(\gamma_{1}, \gamma_{3}\right.$, and $\left.\gamma_{4}\right)$; heat convects from the sample through a Newton cooling boundary condition on the fourth side $\left(\gamma_{2}\right)$. See Figure 17 . 

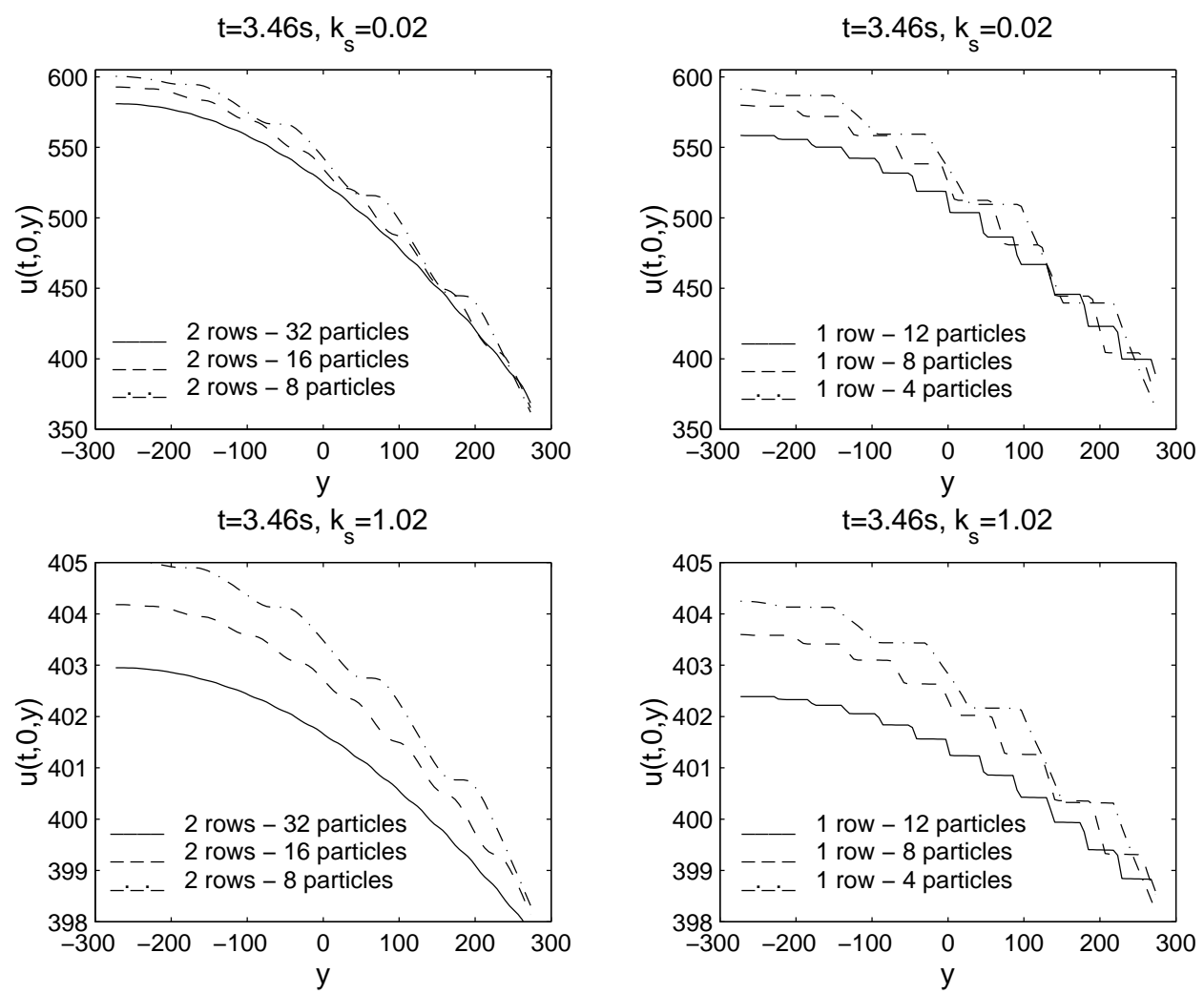

Figure 18: Temperature profiles at $x=0,25 \%$ volume fraction

In the following subsections we investigate the following effects on heat transfer: varying particle size and placement for a fixed volume fraction, and varying volume fraction for a fixed particle size.

\subsection{Fixed Volume Fraction}

Here we investigate the effect of particle size and placement on heat transfer through a heated composite adhesive. We fix the volume fraction and vary the size and placement of the particles. We work with the same six uniform configurations of $25 \%$ volume fraction as in the previous section: 3 samples with particles arranged in two rows, 3 samples with particles arranged in 1 row. See Figure 11 for the different particle arrangements. Again, the filler particles are aluminum $\left(k_{p}=217 W / m K\right)$.

In Figure 18 we plot the temperature profiles through the middle of 


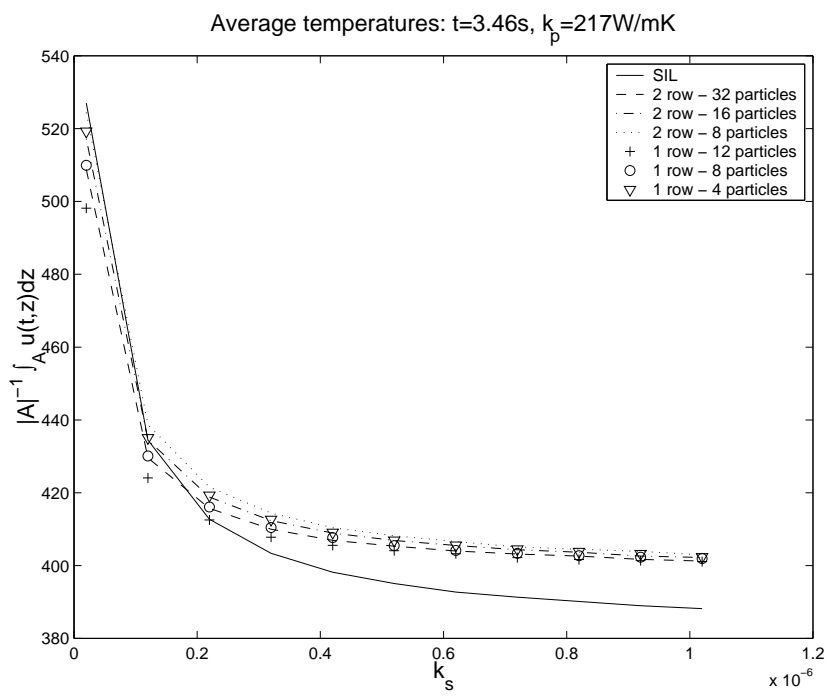

Figure 19: Average temperature for heated slices with $25 \%$ volume fraction

each slice $(x=0)$ at the final time $t=3.46 \mathrm{~s}$ for two different silicone thermal conductivities. The top two plots give temperature profiles for a low silicone conductivity $\left(k_{s}=0.02 \mathrm{~W} / \mathrm{mK}\right)$ slice; the bottom two plots show temperature profiles for a high silicone conductivity $\left(k_{s}=1.02 \mathrm{~W} / \mathrm{mK}\right)$ slice. Temperatures are plotted at the final time, rather than an intermediate time, since there is plenty of heat remaining in the composite slice. The temperature trends are more pronounced than in the pulsed heat source scenario. In both the one row and two row configurations, the smaller, closer particle arrangements result in better heat transfer, i.e., low temperatures. Also, for the eight particle case, heat transfer is more efficient when the particles are aligned in one row as opposed to two rows.

In Figure 19 we plot the average temperature for each of the uniform particle arrangements at the final time $t=3.46 \mathrm{~s}$ across a range of silicone thermal conductivities. Also included is the average temperature in the silicone slice (SIL) at $t=3.46 \mathrm{~s}$ across the same range of conductivities. There are slight differences in the average temperatures between the various uniform geometries of $25 \%$ volume fraction, though the temperature ranges are larger than in previous experiments. In general the composite slices with smaller, more numerous particles produce lower average temperatures. The greatest difference in average temperatures is between the composite 

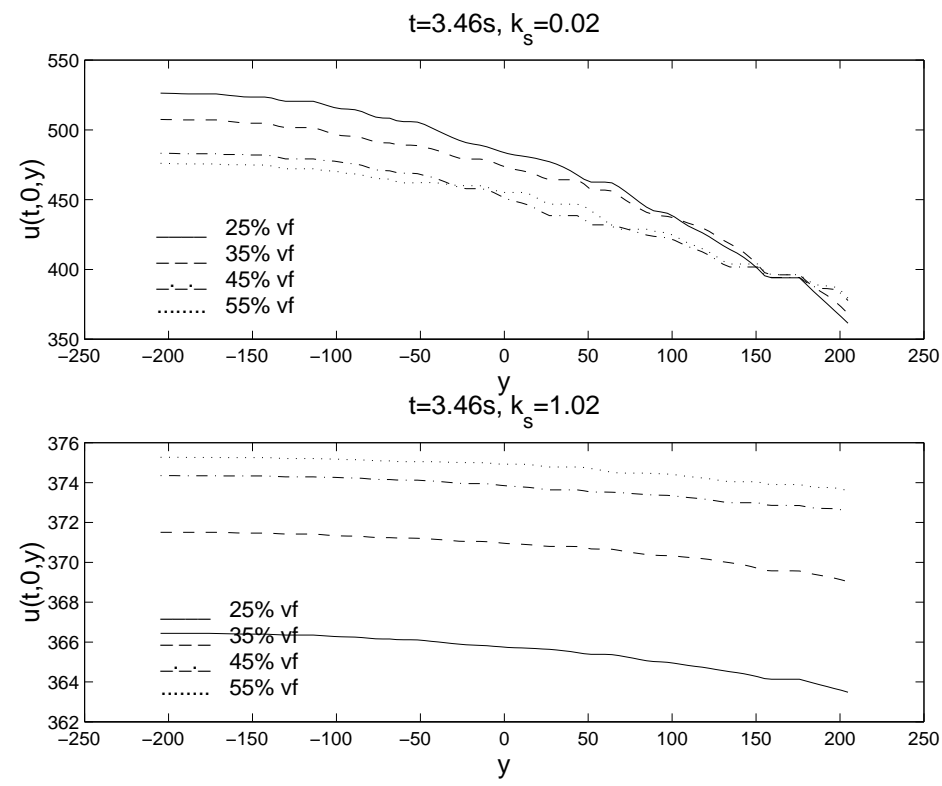

Figure 20: Temperature profiles at $x=0$, varied volume fraction volume fraction

slices and the all silicone slice. With a low silicone thermal conductivity, the composite slices have a lower average temperature than the silicone slice. However, for $k_{s}>0.02 \mathrm{~W} / \mathrm{mK}$, the silicone slice produces a lower average temperature at the final time. This cross-over point depends on the heat transfer coefficient $h$.

The average temperature plot in Figure 19 indicates that, for large silicone conductivity, the silicone slice appears to transfers the heat through the slice more effectively than the composite slices with similar silicone thermal conductivity. This result does not match results from Section 2.

\subsection{Varied Volume Fraction}

In this section we look at the effect of volume fraction on heat transfer through a heated composite adhesive. We fix the particle size $(R=10.50 \mu)$ and vary the number of particles (volume fraction) in the composite adhesive. The particles are aluminum $\left(k_{p}=217 \mathrm{~W} / \mathrm{mK}\right)$ and are arranged randomly to form volume fractions of $25 \%, 35 \%, 45 \%$, and $55 \%$. See Figure 14 for the random particle arrangements. 


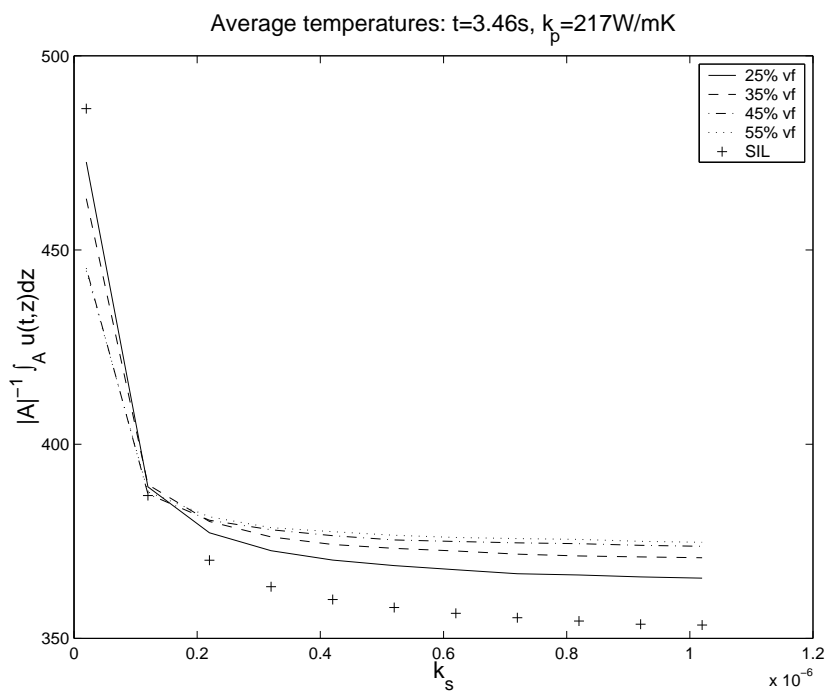

Figure 21: Average temperatures, varied volume fraction

Again, we look for trends in heat transfer as we increase the volume fraction. In Figure 20 we plot the temperature profiles through the middle of each slice $(x=0)$ at the final time $t=3.46 \mathrm{~s}$ for two different silicone thermal conductivities. The top plot displays temperature profiles for the low silicone conductivity $\left(k_{s}=0.02 \mathrm{~W} / \mathrm{mK}\right)$; the bottom plot displays temperature profiles for the high silicone conductivity $\left(k_{s}=1.02 \mathrm{~W} / \mathrm{mK}\right)$. Note that with a low silicone conductivity, the larger volume fractions result in lower temperatures through the middle of the slice. The total temperature difference between profiles, from $25 \%$ to $55 \%$ volume fraction at the boundary $\gamma_{4}$, is approximately $50 \mathrm{~K}$. The reverse behavior is true in the case of a high silicone thermal conductivity: larger volume fractions result in higher temperatures through the middle of the slice. The temperature difference at $\gamma_{4}$ is only $10 \mathrm{~K}$.

Again, we see that with a high silicone thermal conductivity, the silicone slice appears to be more efficient extracting the heat from the slice. We refer to this phenomena as the speed bump behavior, i.e., the particles in the composite slice act as speed bumps and slow down heat transfer. In Figure 21 we plot the average temperature for each composite slice and the silicone slice at the final time $t=3.46 \mathrm{~s}$ across a range of silicone thermal conductivities. With a large silicone thermal conductivity $\left(k_{s}>0.1 \mathrm{~W} / \mathrm{mK}\right)$, larger volume fractions result in higher average temperatures. 


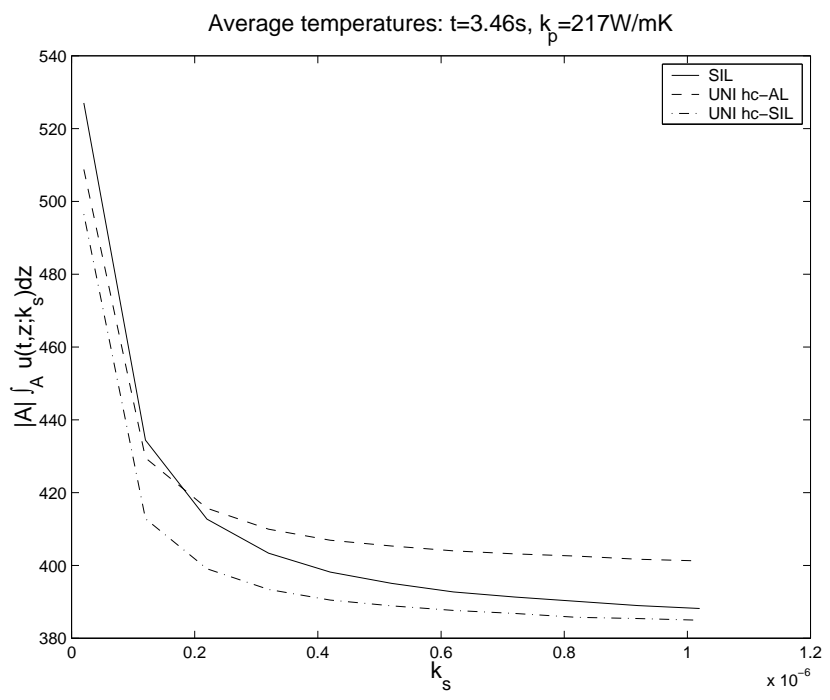

Figure 22: Average temperatures, comparing heat capacity

\subsection{The Speed Bump Behavior}

The speed bump behavior seems to indicate that, at the higher silicone conductivity range, it is more efficient to not add particles. In other words, the highly conductive particles hinder, rather than aid, heat dispersion. This, of course, is not the case. The speed bump behavior is due to the different volumetric heat capacities of each slice.

The all silicone slice has lower temperatures at the final time due to the fact that the composite slice has a larger volumetric heat capacity $\left(\rho c_{p}\right)$. Though starting out at the same temperature, the slice with particles stores more heat (energy) than the all silicone slice. In the previous pulsed and heated slice experiments, the aluminum particles have a heat capacity of $\rho c_{p}=2.43 \times 10^{6} \mathrm{~J} / \mathrm{m}^{3} \mathrm{~K}$ whereas the silicone has a heat capacity of $\rho c_{p}=1.55 \times 10^{6} \mathrm{~J} / \mathrm{m}^{3} \mathrm{~K}$. If the aluminum particles had the same heat capacity as the silicone (i.e., same stored energy), then the composite slice with highly conductive particles should be as or more efficient at dissipating heat than the silicone slice.

To test this we adjusted the heat capacity of the aluminum particles. In Figure 22 we plot the average temperatures at the final time $t=3.46 \mathrm{~s}$ across a range of silicone thermal conductivities for a silicone slice (SIL), and two composite slices each with a uniform aluminum particle arrangement of 
$25 \%$ volume fraction. One composite slice (UNI hc-AL) has the particle heat capacity of aluminum (i.e., $\rho=2.7 e-12 g / \mu^{3}, c_{p}=0.90 \mathrm{~J} / g K$ ), the other composite slice (UNI hc-SIL) has the particle heat capacity of silicone (i.e., $\left.\rho=1 e-12 g / \mu^{3}, c_{p}=1.55 J / g K\right)$. As expected, the composite slice containing particles with the heat capacity of silicone has lower average temperatures than the all silicone slice. In other words, the presence of highly conductive particles does indeed aid the heat transfer.

\section{Continuous Heat Source Experiments}

In this section we again consider the basic model presented in Section 1, however we use a continuous heat source at the boundary $\gamma_{4}$ rather than a laser pulse heat source. The heat source is a constant flux term of the form $S_{0}(t)=S_{c}$. The sides $\gamma_{1}$ and $\gamma_{3}$ are insulated, and $\gamma_{2}$ has a Newton cooling boundary condition. See Figure 2 for the pulsed heat source experimental setup.

For completeness and comparison purposes, we again investigate the following effects on heat transfer: varying particle size and placement for a fixed volume fraction, and varying volume fraction for a fixed particle size. To this end, we consider the steady state solutions of system (1). This is obtained, for all runs, after a time integration of 100 seconds.

\subsection{Fixed Volume Fraction}

We fix the volume fraction and vary the size and placement of the aluminum particles. See Figure 11 for the six different uniform particle arrangements.

In Figure 23 we plot the temperature profiles through the middle of each slice $(x=0)$ at the time $t=100 \mathrm{~s}$ for two different silicone thermal conductivities. The top plot displays temperature profiles for a low silicone conductivity $\left(k_{s}=0.02 \mathrm{~W} / \mathrm{mK}\right)$ slice; the bottom plot displays temperature profiles for a high silicone conductivity $\left(k_{s}=1.02 \mathrm{~W} / \mathrm{mK}\right)$ slice. The temperature differences are slight, still the composite slices with smaller, closer particles obtain lower steady state temperatures through the slice middle. Also, the slices with particles in one row versus two rows tend to channel the heat more efficiently, resulting in slightly lower steady state temperatures through the slice middle.

The same trends are observed with the average temperatures for various silicone conductivities. In Figure 24 we plot the average temperature for each of the uniform particle arrangements and the corresponding silicone slice at the final time $t=100 \mathrm{~s}$ across a range of silicone thermal conductivities. Note 

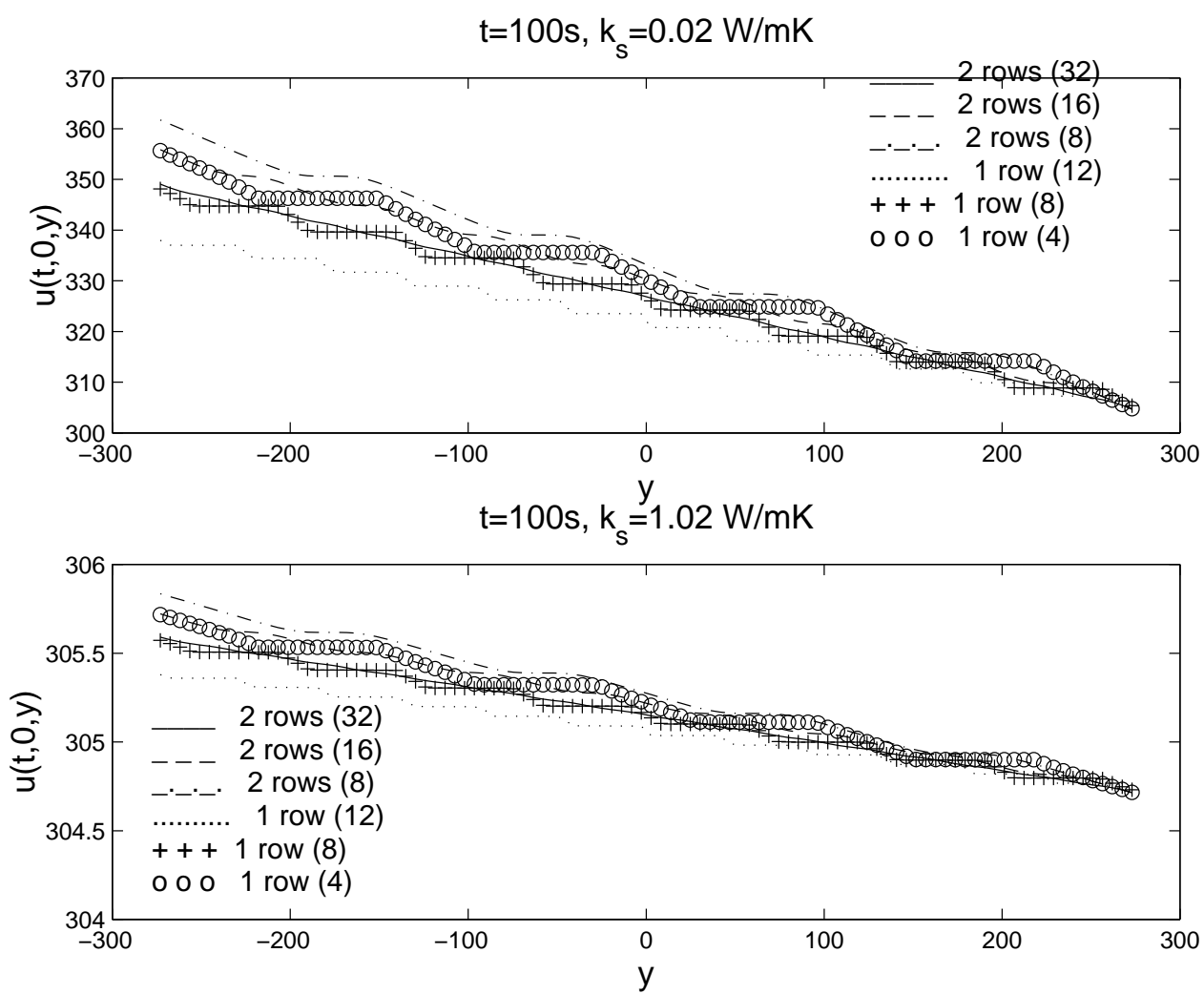

Figure 23: Temperature profiles at $x=0,25 \%$ volume fraction 


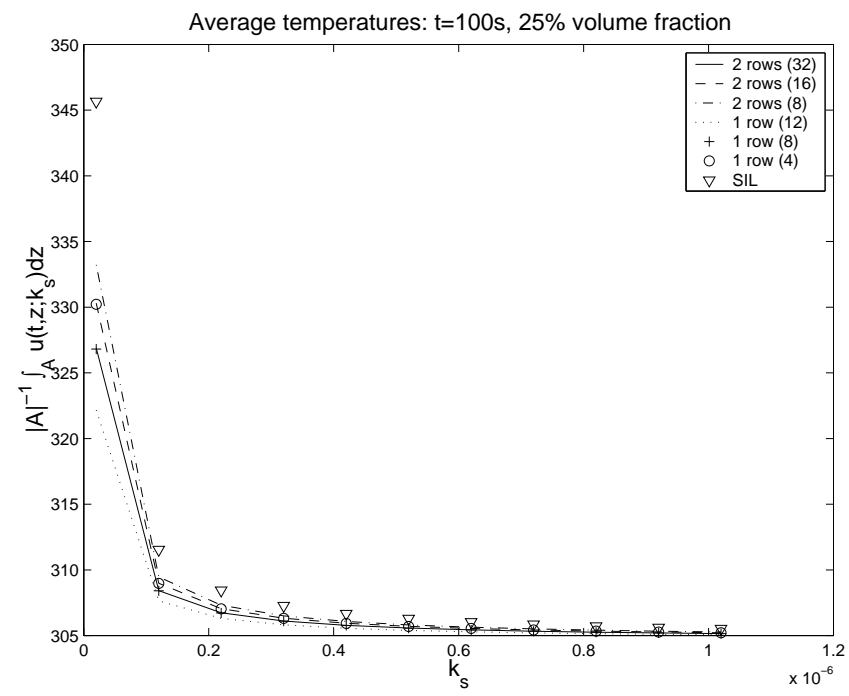

Figure 24: Average temperatures for slices with a continuous heat source

that the range of average temperatures is larger than for the case of a pulsed heat source (compare with Figure 19). With the lowest silicone conductivity, average temperature differences between silicone and composite slices are as much as $20 K$. With a high silicone conductivity, the average temperature difference drops to $1 K$.

\subsection{Varied Volume Fraction}

In this section we look at the effect of volume fraction on heat transfer through a composite adhesive with a continuous heat source. The particle size is fixed $(R=10.50 \mu)$, the volume fraction varies from $25 \%$ to $55 \%$. The particles are aluminum $\left(k_{p}=217 \mathrm{~W} / \mathrm{mK}\right)$ and arranged randomly. See Figure 14 for the random particle arrangements.

In Figure 25 we plot the temperature profiles through the middle of each slice $(x=0)$ at the final time $t=100 \mathrm{~s}$ for two different silicone thermal conductivities. The top plot displays temperature profiles for a low silicone conductivity $\left(k_{s}=0.02 \mathrm{~W} / \mathrm{mK}\right)$ slice; the bottom plot displays temperature profiles for the high silicone conductivity $\left(k_{s}=1.02 \mathrm{~W} / \mathrm{mK}\right)$ slice. With both conductivities, the slices with larger volume fractions obtain lower temperatures through the middle of the slice. We obtain similar results as the pulsed heat source scenario; compare with Figure 20. 

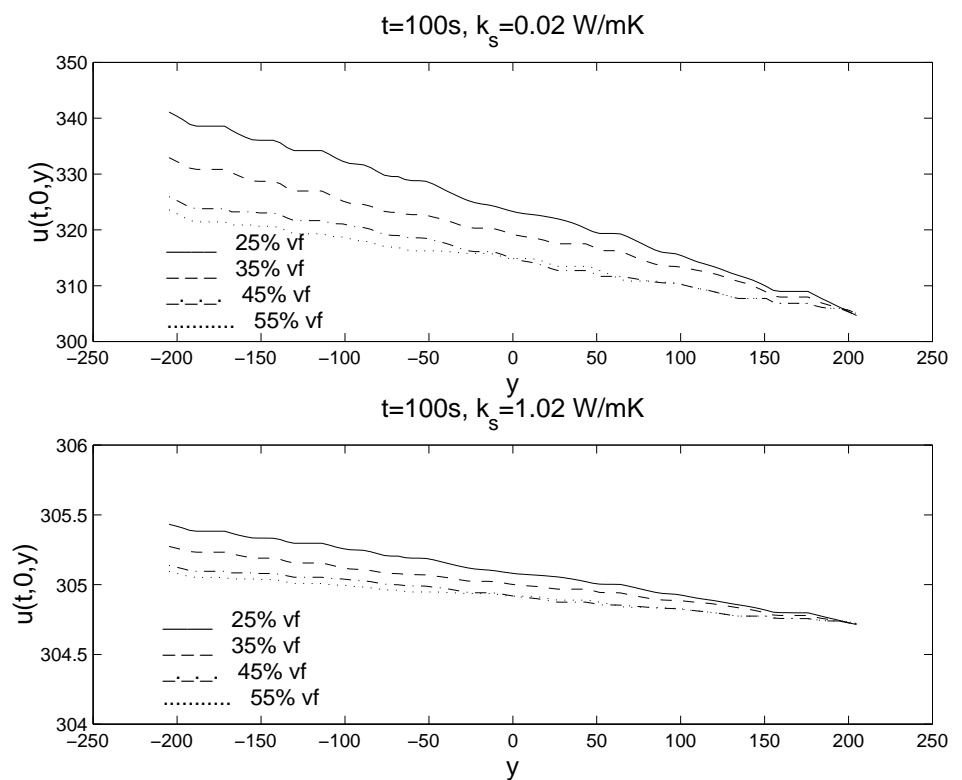

Figure 25: Temperature profiles at $x=0$, varied volume fraction

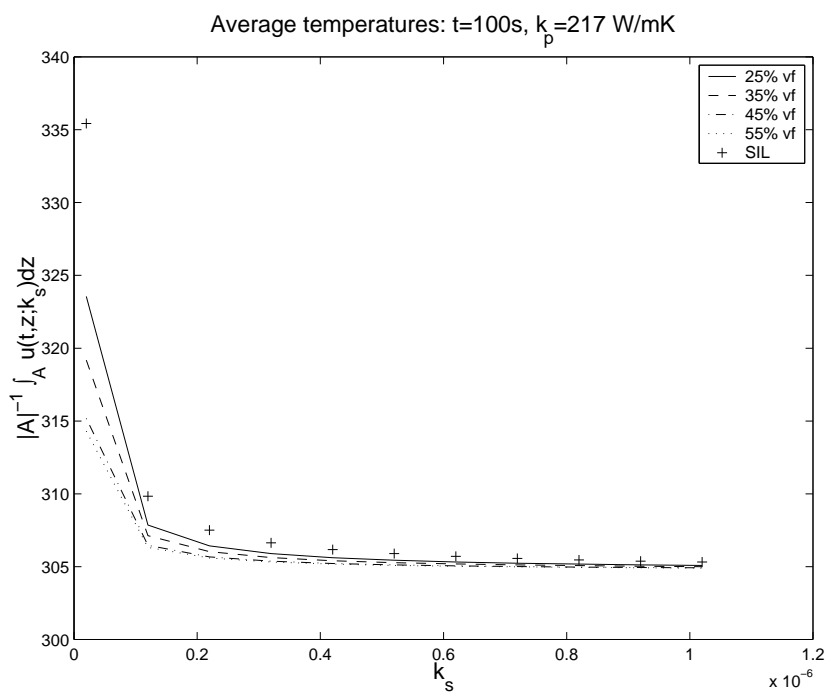

Figure 26: Average temperatures, varied volume fraction 
Finally, in Figure 26 we plot the steady state average temperatures for each of the volume fraction slices, and the all silicone slice, at the final time $t=100 \mathrm{~s}$ across a range of silicone thermal conductivities. The trend is the same as in the pulsed heat source scenario: the larger the volume fraction the lower the average temperature. Additionally, all the composite slices obtain a lower average temperature than the all silicone slice for the full range of silicone thermal conductivities.

\section{Summary}

We investigated the heat transfer through composite adhesives under three experimental scenarios: a laser pulse heat source, a preheated sample, and a continuous heat source. In the pulse heat source scenario, we reported on a variety of results (average flux, average sensitivity) to better understand the basic heat transfer behavior. Similar results are available for the preheated and continuous heat source scenarios as well.

For all three scenarios we considered the effect of particle size and placement for a fixed volume fraction as well as the effect of volume fraction for a fixed particle size. Results for the continuous and pulse heat sources were similar. Namely, smaller, closer particles are slightly more effective at transferring heat through a composite slice. Composites with higher volume fractions are also more effective. The effectiveness, however, depends on the thermal conductivity of the silicone. In other words, with a large silicone thermal conductivity, these effects become less pronounced. Alternatively, when working with composites with low silicone thermal conductivities, the heat transfer may be improved by slightly increasing the silicone thermal conductivity.

These conclusions apply to the preheated scenario as well, but are not evident in the temperature profiles and average temperature results displayed in Section 3. We informally called this discrepancy the speed bump behavior. It is due to the fact that the preheated composite slices have a larger volumetric heat capacity than an all silicone slice. In other words, more heat is stored and transfered in the composite slices. Similarly, larger volume fraction slices have a larger volumetric heat capacity.

\section{Acknowledgments}

This research was supported in part (H.T.B. and S.W.) by the U.S. Air Force Office of Scientific Research under grant AFOSR F-49620-00-1-0026. 


\section{References}

[1] HT Banks and KL Bihari "Analysis of thermal conductivity in composite adhesives", CRSC Tech. Report TR01-20, NC State Univ. August 2001; Func. Anal. and Opt., to appear. 